\title{
Scalable parallel computation of explosively formed penetrators (EFPs)
}

\author{
E. L. Baker, C. Chin, B. E. Fuchs \& J. Pham \\ Energetics and Warheads Division, U.S. Army, ARDEC, Picatinnyl, \\ $N J, U S A$
}

\begin{abstract}
Under the DoD High Performance Computer Modernization Program, the ARDEC Energetics and Warheads Division has successfully developed and demonstrated scalable parallel warhead mechanics modeling of an advanced explosively formed penetrator (EFP) warhead device. Warhead development commonly uses advanced computer modeling which can take extreme amounts of computer time. The recent application of scalable parallel computers has demonstrated the capability to achieve approximately a 120 times speed-up of EFP warhead modeling. Speed-ups were demonstrated using modeling of a copper lined EFP anti-armor warhead using the PCTH computer program on a number of multiple processor computers including a 256 node IBM SP2, 128 node Silicon Graphics Origin 2000 and smaller multiple processor Silicon Graphics workstations. This pioneering work clearly demonstrates the feasibility of applying parallel computers for warheads development.
\end{abstract}

Keywords: explosively formed penetrator, scalable computing, parallel computing.

\section{Background}

Continuum simulation of high rate explosive applications using finite element, finite difference and discrete particle methodologies have received considerable attention since the advent of digital computers (Von Neumann and Richtmyer [1], Mader [2], Wilkins [3]). Explicit high rate continuum simulation is extremely computationally intensive. As a result, less computationally demanding analytic models of high explosive systems have been widely developed and used (Baker [4], Yates et al [5]). However, analytic models rely 
on many simplifying approximations and empirical relationships. Continuum modeling techniques allow the inclusion of advanced material descriptions and attempt to simulate detailed distributed material responses. The resolution of detailed wave dynamics and highly path dependant nonlinear material response are often very important aspects of high explosive application simulations that simply cannot be reasonably approximated using simplified analytic modeling. As computational capabilities have progressed, so has the application of continuum simulations of high explosive applications including shaped charge and explosively formed penetrator (EFP) warheads.

\section{Warheads modeling}

Previous efforts have demonstrated the successful application of explicit high rate finite difference and finite element computer modeling to the simulation and design of shaped charges (Baker and Campbell [6]) and EFPs (Baker and Liu [7], Fong et al [8]). This modeling has been primarily axisymmetric, as the initial geometries are often axisymmetric. Figure 1 presents an axisymmetric shaped charge simulation calculated using CALE. Figure 2 presents an axisymmetric EFP simulation calculated using DYNA2D. CALE (Tipton [9]) is an arbitrary Lagrangian Eulerian finite difference program. DYNA2D (Hallquist [10]) is a Lagrangian finite element program.

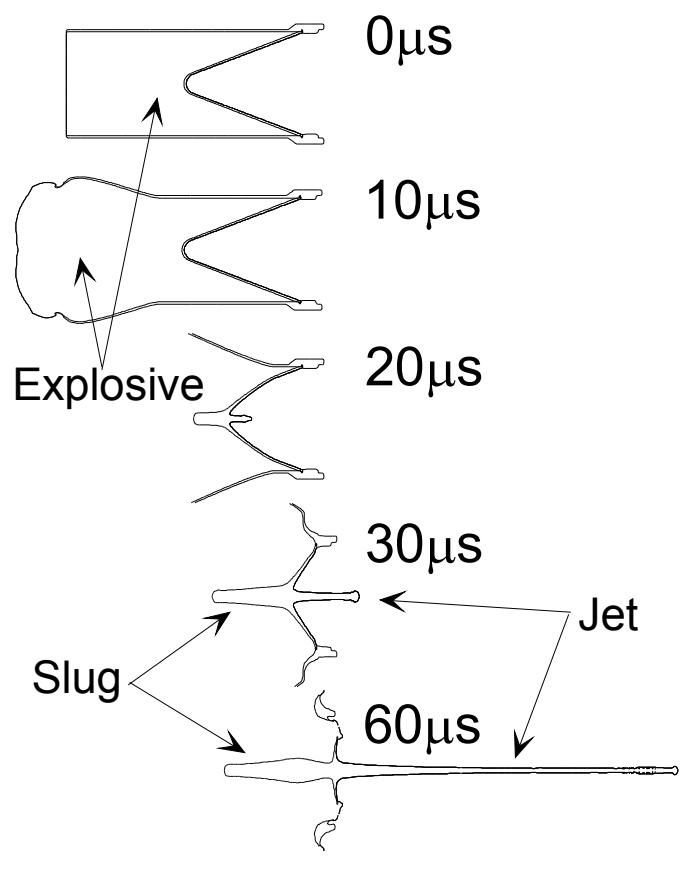

Figure 1: Axisymmetric shaped charge simulation. 

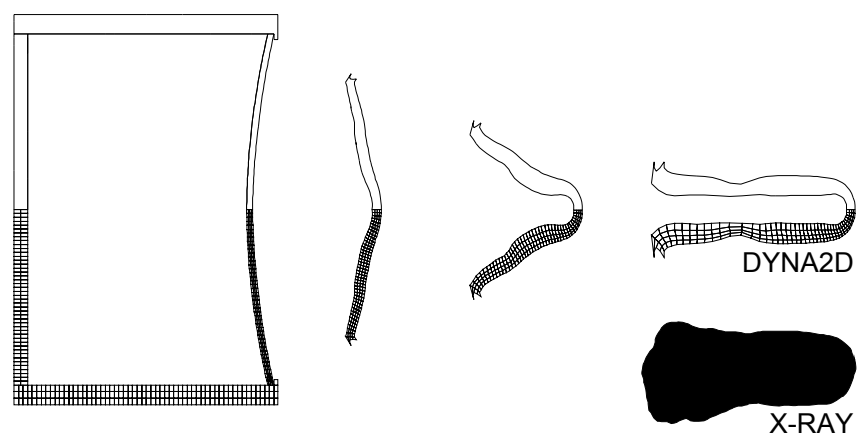

Figure 2: Axisymmetric EFP simulation and experimental flash x-ray.

The liner collapse and jet formation of axisymmetric shaped charges are normally considered to be a primarily axisymmetric event. In contrast, the folding of an EFP liner into a desirable projectile geometry is inherently three dimensional, as the liner normally buckles during the folding process. This buckling behavior results in a final projectile configuration with buckling at the rear flare section. This means that accurate modeling of EFP warheads should include full three dimensional continuum simulation. Three dimensional continuum simulation is at least an order of magnitude more intensive than two dimensional simulation. As a result, full three dimensional explicit continuum simulation requires considerable computer resources. In particular, Eulerian finite difference modeling has historically been extremely computationally intensive, but often required in order to resolve highly distorted geometries or eroding material that cannot be resolved using traditional Lagrangian methodologies.

\section{Scalable software}

Under the DoD High Performance Computer Modernization Program, Common High Performance Computing Software Support Initiative (CHSSI) a scalable parallel version of the $\mathrm{CTH}$ Eulerian high rate finite difference program (McGlaun et al [11]) was developed. This scalable software suite is for the simulation of multidimensional, multi-material, large deformation, strong shock wave propagation problems in solid mechanics. As a part of this development, the ARDEC Energetics and Warheads Division is assessing the performance and feasibility of performing parallel modeling for the design and development of warheads technology. A part of this assessment includes benchmarking of warheads demonstration calculations.

\section{Scalable EFP calculations}

The ARDEC Energetics and Warheads Division has a long history of warheads design and technology development. A part of that technology development includes EFP warheads. As a result, a number of baseline EFP designs have 
been developed for general investigations and applications. One of these baseline designs was used for a computational comparison to assess the performance and feasibility of performing parallel EFP modeling for the development of warheads technology. This relatively conservative copper lined EFP warhead produces a final projectile with length to diameter ratio of about three. A demonstration calculation was run on the 128 node U.S. Army ARL Silicon Graphics Origin 2000 and the 256 node U.S. Air Force Aeronautical Systems Center IBM SP2. Figure 3 presents a visualization of the three dimensional EFP simulation. The three dimensional liner buckling is clearly evident during the formation of the projectile flare. The flare is desirable for aerodynamic stability of the projectile. In addition, the liner edge erosion material process is clearly evident. Figure 4 presents the three dimensional EFP simulation final shape. The final buckling of the flare region is clearly evident. Figure 5 presents an experimental streak photograph of the EFP taken at the ARDEC small scale test area. The experimental result clearly shows the three dimensional buckling on the projectile flare, as well as the erosion material debris. The simulation agreement to experiment is excellent.

\section{Computational performance}

Run time comparison using varied number of CPUs was conducted for scalable computational performance benchmarking. Although the simulation was run to completion on both the Silicon Graphics Origin 2000 and the IBM SP2, the benchmarking was done with a shorter run time in order conserve computer time. The calculated speed-ups on the Silicon Graphics Origin 2000 are presented on Table 1. The calculated speed-ups on the IBM SP2 are presented on Table 2. Based on the results, both machines can be expected to achieve computational speed-ups of about 120 times using 1024 processors. This is a considerable increase, as current warheads calculations are typically completed at ARDEC using a single processor of a Silicon Graphics Origin server using the same processor and processor speed as the ASC Origin 2000.

Table 1: $\quad$ Scalable computational speed-up comparison for the Silicon Graphics Origin 2000.

\begin{tabular}{|c|c|}
\hline \multicolumn{2}{|c|}{ Silicon Graphics Origin 2000 } \\
\hline \# CPUs & Speed Up \\
\hline \hline 1 & 1.0 \\
\hline 4 & 1.5 \\
\hline 8 & 2.1 \\
\hline 32 & 5.6 \\
\hline 1024 & $119.5^{*}$ \\
\hline
\end{tabular}

$*=$ Projected 


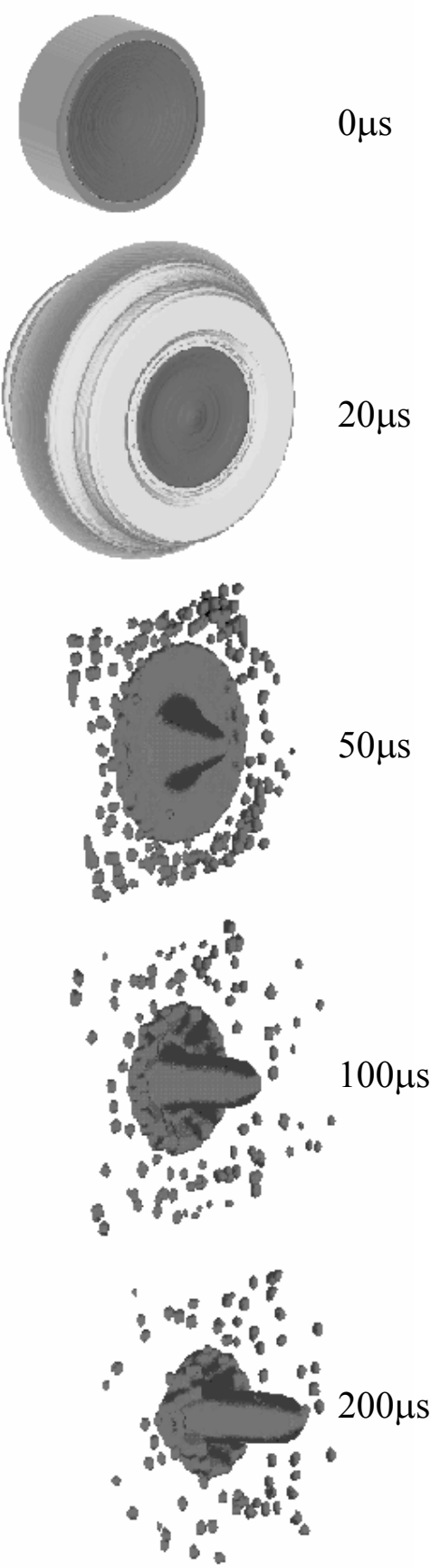

Figure 3: Three dimensional EFP simulation. 


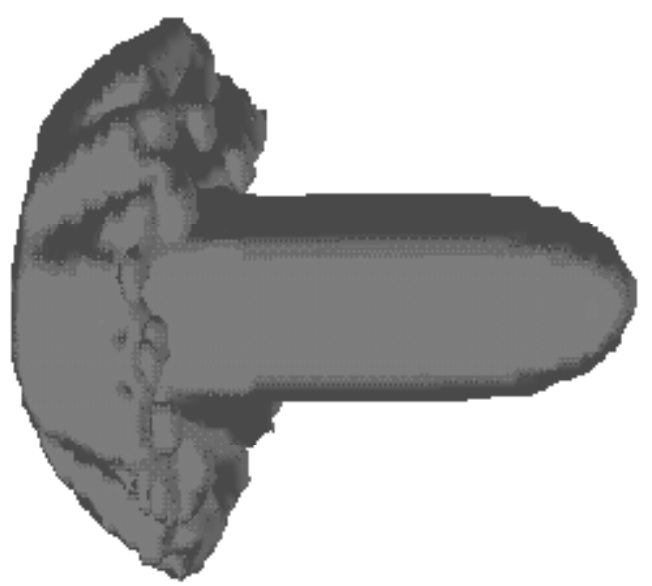

Figure 4: Three dimensional EFP simulation final projectile shape.

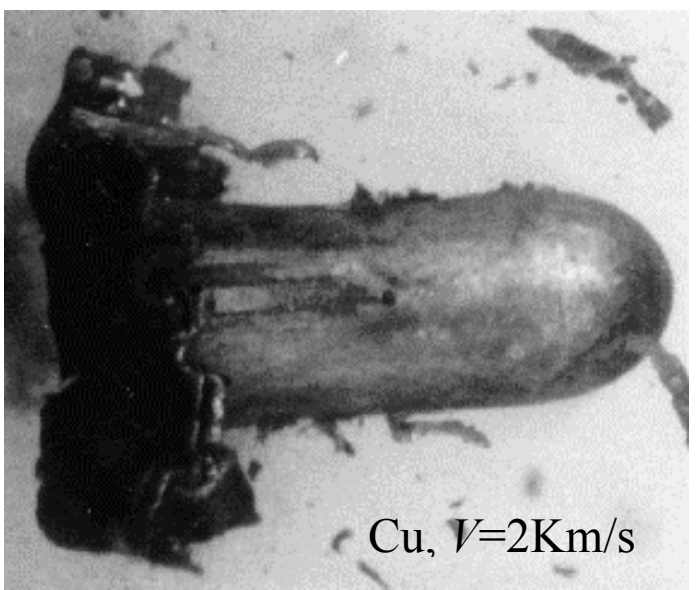

Figure 5: Experimental streak photograph of the same EFP.

Table 2: $\quad$ Scalable computational speed-up comparison for the IBM SP2.

\begin{tabular}{|c|c|}
\hline \multicolumn{2}{|c|}{ IBM SP2 } \\
\hline \# CPUs & Speed Up \\
\hline \hline 1 & 1.0 \\
\hline 8 & 1.1 \\
\hline 16 & 2.0 \\
\hline 32 & 3.9 \\
\hline 1024 & $121.7^{*}$ \\
\hline
\end{tabular}

*=Projected 


\section{Conclusions}

The ARDEC Energetics and Warheads Division have successfully developed and demonstrated scalable parallel warhead mechanics modeling of an advanced EFP using the scalable parallel CTH computer program. This recent application of scalable parallel computers has demonstrated approximately that a 120 times computational speed-up would be expected in a 1024 node machine using the current scalable CTH software on either a Silicon Graphics Origin 2000 or an IBM SP2. This pioneering work clearly demonstrates the feasibility of applying parallel computers for warheads development. Ongoing efforts are concentrating on modeling of highly computationally intensive continuum simulation of three dimensional shaped charge behavior.

\section{Acknowledgement}

This work was supported in part by a grant of HPC time from the DoD HPC Centers.

\section{References}

[1] Von Neumann, J. and R.D. Richtmyer. 1950. J. Appl. Phys. 21, 232.

[2] Mader, C.L. 1964. "The Two-Dimensional Hydrodynamic Hot Spot." Los Alamos Scientific Laboratory report LA-3077.

[3] Wilkins, M.L. 1965. "The Use of One- and Two-Dimensional Hydrodynamic Machine Calculations in High Explosive Research." Proceedings of the Fourth Symposium (International) on Detonation, Naval Ordnance Laboratory, White Oak, Silver Spring, MD (October).

[4] Baker, E.L. 1991. "An Application of Parametric Optimization to Analytical Shaped Charge Modeling." Proceedings of the SCS 1991 Eastern Multiconference, Ballistics Conference, New Orleans, LA (April).

[5] Yates, J.E., M.E. Majerus and R.E. Brown. 1995. "A Panel Code Model of the Shaped Charge Liner Collapse Process." Proceedings of the $15^{\text {th }}$ International Symposium on Ballistics, Jerusalem, Israel (May).

[6] Baker, E.L. and R. Campbell. 1993. "Optimized Wave Shaping of a Molybdenum Conical Lined Shaped Charge." Proceedings of the SCS 1993 Eastern Multiconference, Ballistics Conference, Arlington, Va., pp. 3-6 (March).

[7] Baker, E.L., and Liu, A.Y. 1995. "Numerical Optimization of Tantalum Material Properties for Two-Dimensional Lagrangian Modeling of a TNAZ Loaded EFP." Proceedings of the 6th TACOM Combat Vehicle Survivability Symposium, Monterey, CA (March).

[8] Fong, R., B. Rice and W. Ng. 1998. "Hydrocode 3D Simulation of NonAxiSymmetric (NAS) Explosively Formed Penetrator (EFP) Warhead." Proceedings of the $17^{\text {th }}$ International Symposium on Ballistics, Midrand, South Africa (March). 
170 Computational Ballistics III

[9] Tipton, R. 1991. “CALE User's Manual.” CALE Documentation, Lawrence Livermore National Laboratory, Livermore, CA, (July).

[10] Hallquist, J.O. 1984. “User's Manual for DYNA2D -- An Explicit TwoDimensional Hydrodynamic Finite Element Code with Interactive Rezoning." Lawrence Livermore National Laboratory Report UCID18756, Rev. 2.

[11] McGlaun, J.M., Thompson, S.L., and Elrick, M.G. 1990. “CTH: A Three-Dimensional Shock Wave Physics Code." Int. J. Impact Engineering, Vol. 10, pp. 351-360. 